\title{
Development and Psychometric Testing of the Hemodialysis Symptom Distress Scale (HSD-22) to Identify the Symptom Cluster by Using Exploratory Factor Analysis
}

\section{Mei-Chu Chen}

Chang Gung Memorial Hospital Kaohsiung Branch

Chiu-Chu Lin ( $\square$ chiuchu@kmu.edu.tw)

Kaohsiung Medical University https://orcid.org/0000-0002-8947-467X

Ya-Fang Ho

China Medical University

Chia-Chen Wu

Fooyin University School of Nursing

\section{Li-Chueh Kuo}

Chang Gung Memorial Hospital Kaohsiung Branch

\section{Research}

Keywords: Exploratory Factor Analysis, Hemodialysis, Nursing, Scale, Symptom Distress, Symptom Cluster

Posted Date: July 22nd, 2020

DOI: https://doi.org/10.21203/rs.3.rs-42010/v1

License: (c) (i) This work is licensed under a Creative Commons Attribution 4.0 International License.

Read Full License

Version of Record: A version of this preprint was published at BMC Nephrology on April 12th, 2021. See the published version at https://doi.org/10.1186/s12882-021-02337-7. 


\section{Abstract}

Aims: To develop a theoretical and psychometrical reliable measurement tool to identify the symptom clusters of patients undergoing hemodialysis (HD).

Design: A cross-sectional descriptive study.

Methods: To examine the psychometric properties of the HD symptom distress (HSD) scale, 216 subjects were recruited from a HD center of medical university hospital in Southern Taiwan from February, 2019 to April, 2019. Construct validity was evaluated by exploratory factor analysis (EFA), and the internal consistency and test-retest reliability were estimated by Cronbach's alpha and intraclass correlation coefficient (ICC).

Results: The HSD scale was composed of five factors with 22 items, including insufficient energy/vitality, cardiac-pulmonary distress, sleep disturbance, musculoskeletal distress, and gastrointestinal distress, with factor loading ranging from 0.62 to 0.87 , explaining $65.5 \%$ of the total variance. Cronbach's alpha coefficient of the HSD total scale was 0.93 , and five subscales ranged from 0.73 to 0.87 . The test-retest reliability was $0.92(p<0.001)$ by using the intraclass correlation coefficient (ICC) for HSD-22 scale.

Conclusion: Results indicated that the HSD-22 scale had initial satisfactory validity and reliability. Therefore, this tool can be used to identify the symptom clusters of patients receiving HD.

Impact: Patients receiving HD often experienced multiple symptoms concurrently and may impact their quality of life. A valid and reliable tool is needed to assess the symptom distress of patients receiving HD in terms of the perspective of symptom clusters. Although many studies had explored symptom clusters related to patients receiving $\mathrm{HD}$, the clusters form had problems with overlapping, vagueness, lack of cluster-specific, and difficulty in discerning their common mechanism under the cluster. Psychometric testing from our study indicated that the HSD-22 scale can be employed to identify the symptom clusters of patients receiving HD in the clinical setting. Such identification enables healthcare professionals provide interventions to release patients' symptom distress efficiently.

\section{Introduction}

In accordance with the United States Renal Data System (USRDS) Report, the global population of endstage kidney disease (ESKD) patients had increased by nearly $20 \%$ since 2000 , and the prevalence of ESKD patients who received renal replacement therapy with hemodialysis (HD) in the US increased more than $80 \%$ from 2000 to 2017 [1]. The prevalence of ESKD patients in Taiwan from 2003 to 2016 was increased $71.8 \%$, with 3,251 per million population undergoing dialysis in 2016 , ranking the top one in the world; it seemed an increasing trend year by year. It became a global [2]. health issue in nursing care for patients receiving dialysis. 
HD was the primary treatment for patients with ESKD in Taiwan, although HD was a life-saving treatment, but a considerable number of patients suffered from multiple symptoms distress due to quality of life reduced $[3,4]$. The interventions for symptom distress in clinical practice were often focused on specific symptom, but increased evidences indicated that symptoms of patients with ESKD occurred in group concurrently, it so-called symptom clusters. Therefore, it had great value to develop a measurement tool to identify the symptom clusters of patients undergoing HD.

\subsection{Background}

Patients with ESKD on dialysis suffered from a high symptom burden because of the disease itself, the treatment, and comorbid conditions, thereby leading to poor quality of life [5]. The multiple symptoms experienced by patients receiving HD were reported including tiredness, sleep disturbance, dry mouth, muscle weakness, and pruritus [3, 4], insomnia, nausea, anorexia, pruritus, and shortness of breath [6]. More specifically, Fidan et al. reported that almost all patients receiving HD had one or more musculoskeletal problems; the most common was muscle cramps, myalgias and arthralgias [7]. According to the reports of 32 participants across three focus groups and 87 survey respondents, the most common physical distress, and symptoms experienced by patients receiving HD were fatigue, cramping, and body aches [8]. In addition to the physical symptoms, depression, anxiety, worried, and frustrated commonly occurred in patients on HD [7-11]. Whether or not the psychological symptoms primarily resulted from the physical symptoms, it needed to be further clarified to identify the independent clusters.

The interventions of symptom distress in clinical practice were primarily focused on a specific symptom. However, it had increasing evidence to support that symptoms of patients with ESKD occur in group concurrently, it was so-called symptom clusters. Although studies had explored symptom clusters related to patients receiving HD [10,12], the clusters formed had problems with overlapping, vagueness, and lacked of cluster-specific, it was difficult in discerning their common mechanism under the cluster. A valid and reliable tool is needed to assess the symptom distress of hemodialysis patients in terms of the perspective of symptom cluster.

\section{The Study Method}

\subsection{Aim}

The aim of this study was to develop a theoretical and psychometrical reliable measurement tool to identify the symptom clusters of patients undergoing HD.

\subsection{Design}

A cross-sectional descriptive study was performed in this study.

\subsection{Instrument Development}




\subsubsection{Item generation}

To generate an item pool, we had referred to previous literature [10,12-15]. Based on the existing literature and the experiences from patients receiving HD, 26 candidate items were generated to form an initial draft of the hemodialysis symptom distress (HSD) scale.

\subsubsection{Determination of content validity}

After the pool of candidate items had been developed, its content validity was tested by five experts including one nephrologist, two nurse educators, and two nurse practitioners working in a HD center of medical university hospital, three of them with a PhD and specialized in instrument development and nephrology. These experts using a four-point Likert scale to rate the relevance and wording of each item, with scores as follows: 1-least relevant, 2-somewhat relevant, 3-quite relevant and 4-most relevant. If an expert rated any item $<4$, the expert was asked to provide his/ her suggestion for the item modification.

We used the content validity index $(\mathrm{CVI})$ to quantify the extent of expert agreement. The proportion of experts who rated items as 3 or 4 [16] was used to analyze the experts' ratings for relevance and clarity of items. The relevance of symptom distress and accuracy of each item in this initial draft were assessed by five experts. The CVI was 0.89 . Four items with problematic wording were revised based on the recommendations of the panel to result a final draft containing 26 items (i.e., the HSD-26).

\subsubsection{Determination of face validity}

To evaluate the face validity of the HSD scale, investigators administered the draft of the instrument (i.e., the HSD-26) to a convenience sample from the data collection sites in this study. Ten patients receiving HD were invited to a pilot study of HSD-26 for clarity, comprehension and ease of response. Items were scored on a 4-point scale from 1 (never) to 4 (always). Total possible scores ranged from 26-104, with higher scores indicating higher symptom distress.

\subsection{Study Participants}

Participants were recruited from a HD center of medical university hospital located in southern Taiwan which possessed the largest hemodialysis center having approximately 940 patients receiving HD. Participants who met the following criteria were recruited: patient was 20 years of age, had undergoing $\mathrm{HD}$ for $\geq 6$ months, and able to comprehend and communicate by Mandarin or Taiwanese.

Per request of the study investigators, HD nursing staffs referred participants from HD outpatient clinics to investigators for recruitment. Two-hundred and sixteen participants agreed to participate in this study.

\section{Data collection}

After receiving the written informed consent from participants, they filled out the questionnaires during the period of receiving hemodialysis, and data were collected from February to April, 2019.

\subsection{Ethical considerations}


The study was approved by the Chang Gung Memorial Hospital Institutional Review Board (IRB 201801071B0).

\subsection{Data analysis}

EFA was used to identify the factor structure. The Kaiser-Meyer-Olkin (KMO) test for sampling adequacy and Bartlett's test for sphericity were performed and the number of factors to be retained was determined by parallel analysis [17]. Items selected met the following four criteria: (a) factor loading $>0.5$; (b) minimum factor membership of three items; (c) no cross factor loaded items; and (d) conceptual coherence of items with its corresponding factor. Internal consistency was assessed by determining Cronbach's alpha coefficients for the overall scale and subscales. A Cronbach's alpha coefficient $>0.70$ was considered satisfactory [18].

\section{Results}

\subsection{Sample Characteristics}

Two hundred and sixteen participants completed the HSD questionnaire, among them, $44.4 \%$ were male and $55.6 \%$ were female, with an age range of 20 to 88 years (mean $=63.0, S D=12.75$ ). The educational level of the sample was diverse (53.5\% with elementary school or less; $34.0 \%$ with a high school diploma; $12.4 \%$ with a college degree), and the majority of participants (87.2\%) were married.

\subsection{Exploratory Factor Analysis (EFA)}

Using EFA, the factor structure of initial HSD was analyzed with a sample of 216 participants. Factors were extracted by using principal component analysis, the correlation matrix and pairwise deletion method. The KMO measurement of sampling adequacy was 0.90 ; it indicated excellent sampling adequacy and relatively compact patterns of correlation. Such factor analysis should be produced distinct and reliable factors [19]. Bartlett's test of sphericity was significant (chi-square $=2588.812, d f=$ $231, p<0.000$ ), it showed that there were adequate relationships between the variables [19]. Oblique Promax rotation procedures were used as the method of factor rotation, because HSD scale factors were assumed to be correlated. Four items (items 12, 21, 23, and 24) were eliminated from the draft 26-item HSD due to a factor loading $<0.5$. A five-factor solution for the 22 remaining items provided the most meaningful factor pattern and labeled as insufficient energy/vitality, cardiac-pulmonary distress, sleep disturbances, musculoskeletal distress, and gastrointestinal distress, with loading ranging from 0.62 to 0.87 , explaining $65.5 \%$ of the total variance. The loading ranging of five factors were shown in Table 1 and the factor structures were described as following: 
Table 1

Exploratory factor analysis (EFA) results and Cronbach's alpha coefficients.

\begin{tabular}{|c|c|c|c|c|c|c|}
\hline \multirow[t]{2}{*}{ Symptom } & \multicolumn{5}{|c|}{ Factor loading } & \multirow[t]{2}{*}{ Cronbach's alpha } \\
\hline & Factor 1 & Factor 2 & Factor 3 & Factor 4 & Factor 5 & \\
\hline Factor 1 & & & & & & 0.89 \\
\hline Tiredness & 0.87 & & & & & \\
\hline Lack of vitality & 0.82 & & & & & \\
\hline Lack of energy & 0.81 & & & & & \\
\hline Muscle weakness & 0.78 & & & & & \\
\hline Dry mouth/thirst & 0.73 & & & & & \\
\hline Vertigo & 0.63 & & & & & \\
\hline Headache & 0.62 & & & & & \\
\hline Factor 2 & & & & & & 0.85 \\
\hline Chest pain & & 0.84 & & & & \\
\hline Shortness of breath & & 0.83 & & & & \\
\hline Dyspnea & & 0.77 & & & & \\
\hline Chest tightness & & 0.75 & & & & \\
\hline Arrhythmia & & 0.65 & & & & \\
\hline Lack of appetite & & 0.62 & & & & \\
\hline Factor 3 & & & & & & 0.80 \\
\hline Waking in night & & & 0.82 & & & \\
\hline Trouble falling asleep & & & 0.78 & & & \\
\hline Itchy skin & & & 0.75 & & & \\
\hline Factor 4 & & & & & & 0.77 \\
\hline Joint pain & & & & 0.88 & & \\
\hline Sore muscles & & & & 0.80 & & \\
\hline Numbness & & & & 0.78 & & \\
\hline Factor 5 & & & & & & 0.73 \\
\hline
\end{tabular}

Note. Factor 1 = 'insufficient energy/vitality; Factor 2 = cardiac-pulmonary distress; Factor 3 = sleep disturbance; Factor 4 = musculoskeletal distress; Factor 5 = gastrointestinal distress 


\begin{tabular}{|c|c|c|c|c|c|c|}
\hline \multirow[t]{2}{*}{ Symptom } & \multicolumn{5}{|c|}{ Factor loading } & \multirow[t]{2}{*}{ Cronbach's alpha } \\
\hline & Factor 1 & Factor 2 & Factor 3 & Factor 4 & Factor 5 & \\
\hline Vomiting & & & & & 0.87 & \\
\hline Nausea & & & & & 0.80 & \\
\hline Cramps & & & & & 0.66 & \\
\hline Total scale & & & & & & 0.93 \\
\hline
\end{tabular}

Factor 1, insufficient energy/vitality, had seven items, with factor loading ranging from 0.62 to 0.86 , accounting for $41.2 \%$ of the variance. This factor reflected the symptoms of tiredness, headache, muscle weakness, lack of energy and vertigo brought about by the patient's anemia.

Factor 2, cardiac-pulmonary symptoms, had six items, with factor loading ranging from 0.62 to 0.84 , accounting for $7.3 \%$ of the variance. This factor reflected the cardiopulmonary symptoms of chest pain, shortness of breath, dyspnea, chest tightness caused by volume overload.

Factor 3 , sleep disturbances, it contained three items, with factor loading ranging from 0.75 to 0.82 , accounting for $6.7 \%$ of the variance. This factor reflected the trouble falling asleep or waking in the night due to peripheral neuropathy of uremia.

Factor 4, musculoskeletal symptoms, had three items with factor loading ranging from 0.78 to 0.88 , accounting for $5.6 \%$ of the variance. This factor reflected problems such as muscle numbness and joint pain caused by electrolyte abnormalities such as calcium, phosphorus, and potassium.

Factor 5, gastrointestinal distress, had three items with factor loading ranging from 0.65 to 0.88 , accounting for $4.7 \%$ of the variance. This factor reflected the gastrointestinal symptoms of vomiting and nausea caused by abnormal gastric emptying.

\subsection{Reliability}

After factor structure confirmed, the investigators used Cronbach's alpha coefficient to assess the reliability of the total scale and the factor-based subscales. Cronbach's alpha coefficient for the final version of the HSD-22 total scale was 0.93 , and the subscale alpha coefficients ranged from 0.73 to 0.89 . The stability of the HSD-22 over time was assessed by measuring the test-retest reliability over 2-4 weeks. Twenty participants were selected to retest HSD-22 questionnaire, and the test-retest reliability using the intraclass correlation coefficient (ICC) was $0.916(p<0.001)$.

\section{Discussion}


This study identified five factors via EFA. These five factors were insufficient energy/vitality, cardiacpulmonary distress, sleep disturbances, musculoskeletal distress, and gastrointestinal distress. They were similar to the clusters identified by Yu IC, Huang JY and Tsai YF [10] which included energy and sensory discomfort, gastrointestinal (GI) and cardiac-pulmonary symptoms, cardiovascular symptoms, and electrolyte imbalance. However, these four clusters identified by Yu IC, Huang JY and Tsai YF [10] had apparently problems with overlapping (cardiac- pulmonary symptoms, cardiovascular symptoms) and vague dimension (electrolyte imbalance). Furthermore, the characteristics of symptom distress verified in our study were much more similar to those dimensions of energy/vitality, cardiac-related problems, pain/comfort, and gastrointestinal (GI) system proposed by Jablonski's study (2007). Compared to the symptoms clusters verified in our study with those identified by Yu IC, Huang JY and Tsai YF [10] and Jablonski A [12], the primary difference was our study separated sleep disturbances as a factor, due to sleep disturbed result from multiple influencing factors presented in patients receiving HD [20]. These influence factors may be related to certain symptom distress like pain/comfort [12] or sensory discomfort [10]. It may explain why sleep disturbances was not an independent dimension/cluster in the study of Yu IC, Huang JY and Tsai YF [10] and Jablonski A [12].

Factor 1, insufficient energy/vitality, was one of the most troublesome distress among the multiple symptoms experienced by patients received HD, it was also found in the study of Yu IC, Huang JY and Tsai YF [10] and Jablonski A [12]. This symptom distress, insufficient energy/vitality, was directly related to renal anemia due to lack of erythropoietin [21]. In addition, blood loss during hemodialysis and latent gastrointestinal bleeding were also common causes of anemia in patients [22]. When anemia occurred, insufficient numbers of circulating red blood cells were available to transport and release oxygen to tissues, thus, patients were prone to symptoms occurred simultaneously such as vertigo, headache, muscle weakness, tiredness, and lack of vitality [21].

For patients received HD, 'cardiac-pulmonary symptoms' presented in Factor 2 was a common symptom distress, it often resulted from fluid overload. The main reason was patients' poor water control during dialysis sessions. When patients had difficulty to control or restrict their fluid intake, it may lead to excessive weight gain during dialysis sessions (i.e., interdialytic weight gain, IDWG). A poor IDWG often caused hypotension, dry mouth, chest pain, chest tightness, and arrhythmia during dialysis [23]. Moreover, fluid overload may lead to congestive heart failure [24]. which further caused cardiomegaly, resulting in symptom distress such as shortness of breath, dyspnea, bloating and decreased appetite [25]. It explained why the chest pain, shortness of breath, dyspnea, chest tightness, arrhythmia, and lack of appetite were clustered to the dimension of 'cardiac-pulmonary symptoms' after conducting the factor analysis.

The symptoms under factor 3 included waking in the night, trouble falling asleep and itchy skin, those clustered into a factor called 'sleep disturbances'. Patients with ESKD often experienced restless leg syndrome resulted from urotoxic peripheral neuropathy. The patient frequently felt uncomfortable at night or when lying in bed, especially on a quiet night. These feelings included insect crawling, acupuncture, or deep itchiness, those made patient had to keep moving their feet or get up to walk to gain a little relief, it 
resulted an interrupted sleep [26, 27]. In addition, uremic pruritus caused by calcium and phosphorus deposition may be another factor affecting patients' sleep. It was a chronic, uncomfortable symptom and worsened at night, it caused severe negative effect on the patient's sleep [28]. It explained why waking in the night, trouble falling asleep, and itchy skin synthesized into a new dimension called 'sleep disturbances'.

For patients receiving hemodialysis, electrolyte imbalance was a common issue. Calcium and phosphorus imbalance was one of the electrolyte imbalances; it often caused secondary hyperparathyroidism, and patients were prone to complications of renal osteodystrophy [29]. Renal osteodystrophy caused symptoms distress, such as joint pain and muscle weakness; and Hyperkalemia was also a common electrolyte imbalance due to kidney failure. Potassium ion balance was essential for nerve conduction and muscle contraction. Hyperkalemia caused depolarization of skeletal muscle cell membranes and inhibit skeletal muscle excitement; and caused muscle numbness, sore, and weakness in the limbs [30]. Through factor analysis, joint pain, sore muscles, and numbness were clustered to be a new dimension called 'musculoskeletal symptoms'.

Gastroparesis was a distress for patients receiving HD due to autonomic neuropathy; it prolonged the time to empty their stomach and caused discomfort symptoms, such as nausea, vomiting, and lack of appetite [31]. In addition, Patients receiving HD took the medication of phosphorus binders due to renal osteoporosis. This kind of medication often produced gastrointestinal side effects, such as nausea, vomiting, and abdominal pain [31]. Severe nausea and vomiting easily leaded to electrolyte imbalance and caused cramps further. Furthermore, uremic polyneuropathy may be another factor caused patients to cramp; the earliest symptom was muscle cramps in the lower limbs [32]. Therefore, vomiting, nausea, and cramps were synthesized into a factor called gastrointestinal distress.

Cronbach's alpha coefficients for the HSD-22 total scale (0.93) and each of the five subscales (0.770.85 ) indicated that this newly-constructed instrument had a good internal consistency. The results of test-retest analysis showed that the HSD-22 was relatively stable over a 2-4-week period.

Patient receiving Hemodialysis often experienced multiple symptoms that usually occurred concurrently; a single symptom seldom occurred separately. To provide an effective intervention for symptom distress, a psychometrically robust measurement which captured the essence of symptom clusters under a group of symptoms and shared a common etiology or biomechanics were needed. The HSD-22 developed in this study covered five factors via factor analysis. In these five factors, each factor covered a cluster of symptoms which shared a common etiology or biomechanics discussed above. Therefore, we suggested that the HSD-22 was verified and improved one of the symptom clusters by HPs earlier, it is a valid and reliable scale and can provide a useful clinical assessment tool for healthcare professionals (HPs) working in the HD unit to identify possible symptom clusters of patients undergoing HD. To achieve more efficacy treatments, clinical interventions should be considered in terms of the common mechanism of symptom cluster.

\subsection{Limitations}


In our study, participants were recruited from a medical university hospital, even they came from every corner of southern Taiwan, but still restricted to one hospital and they may unable to on behalf of all hemodialysis of the population in Taiwan. And due to the reduced data, we used EFA to analyze the HSD22 for smaller set of variables and to explore the underlying theoretical structure of a phenomenon, it narrowed construct validity. We suggested that future studies can recruit the participants from overall of Taiwan through multiple medical university hospital, and using confirmatory factor analysis (CFA) to test its construction validity. Then, study result may be more definite.

\section{Conclusions}

In conclusion, a better understanding of patients' symptom distress may increase the treatment efficiency and help patient to reach better health outcomes. A psychometrically robust measure captures clusters of distress from multiple and concurrent symptoms encountered in patients received HD is needed.

Psychometric testing from our study indicated that the HSD-22 scale is valid and reliable, we suggest that this assessment tool can be employed to identify the symptom clusters of patients received HD in the clinical setting. Such identification enables HPs efficiently to provide interventions to release patients' symptom distress. More specifically, HPs can empower patients to verify and manage their own discomfort symptoms associated with symptom clusters.

\section{Declarations}

\section{- Ethics approval and consent to participate}

The study was approved by the Chang Gung Memorial Hospital Institutional Review Board (IRB 201801071B0).

\section{- Consent for publication}

The authors give their consent for the article to be published.

\section{- Availability of data and materials}

The datasets used and/or analyzed for this study are available from the corresponding author on reasonable request.

\section{- Competing interests}

The author(s) declared no potential conflicts of interest with respect to the research, authorship and/or publication of this article.

\section{- Funding}


This study was sponsored by a Research Program (CMRPG810011) from Department of Medical Research, Chang Gung Memorial Hospital.

\section{- Authors' contributions}

Mei-Chu Chen: Study conception and design, Data collection, analysis and interpretation, Drafting of the article.

\section{- Acknowledgements}

The author would like to thank Kaohsiung Chang Gung Memorial Hospital for the research budget subsidy and insightful advice, and the Biostatistics Center, Kaohsiung Chang Gung Memorial Hospital for their help on statistical analysis.

\section{References}

1. US Renal Data System 2019 Annual Data Report: Epidemiology of Kidney Disease in the. United States [https://www.usrds.org/2019/view/ USRDS_2019_ES_final.pdf]

2. US Renal Data System 2018 Annual Data Report: End-stage kidney disease (ESKD) in the United States - International comparisons Retrieved from. https://www.usrds.org/2018/view/Default.aspx

3. García-Llana H, Remor E, Del Peso G, Selgas R. The role of depression, anxiety, stress and adherence to treatment in dialysis patients health-related

quality of life: a systematic review of the literature. Nefrologia. 2014; 34:637-657.

4. Lopes AA, Lantz B, Morgenstern H, Wang M, Bieber BA, Gillespie BW, Li Y, Painter P, Jacobson SH, Rayner HC, et al. Associations of self-reported physical activity types and levels with quality of life, depression symptoms, and mortality in hemodialysis patients: the DOPPS. Clin J Am Soc Nephrol. 2014; 9:1702-1712.

5. Tannor EK, Archer E, Kapembwa K, van Schalkwyk SC, Davids MR. Quality of life in patients on chronic dialysis in South Africa: a comparative mixed methods study. BMC Nephrol. 2017; 18:4.

6. Cao Y, Shen J, Li W, Zhang Y, Guo X, Li CS, Zhang Y, Luo X. The symptom trajectories to clinical remission in Chinese patients with unipolar major depressive disorder. Asia Pac Psychiatry. 2016; 8:309311.

7. Fidan F, Alkan BM, Tosun A, Altunoğlu A, Ardıçoğlu Ö. Quality of life and correlation with musculoskeletal problems, hand disability and depression in patients with hemodialysis. Int J Rheum Dis. 2016; 19:159-166. 
8. Flythe JE, Hilliard T, Castillo G, Ikeler K, Orazi J, Abdel-Rahman E, Pai AB, Rivara MB, St Peter WL, Weisbord SD, et al. Symptom Prioritization among Adults Receiving In-Center Hemodialysis. A Mixed Methods Study. Clin J Am Soc Nephrol. 2018; 13:735-745.

9. Zhang M, Kim JC, Li Y, Shapiro BB, Porszasz J, Bross R, Feroze U, Upreti R, Martin D, Kalantar-Zadeh K, Kopple JD. Relation between anxiety, depression, and physical activity and performance in maintenance hemodialysis patients. J Ren Nutr. 2014; 24:252-260.

10. Yu IC, Huang JY, Tsai YF. Symptom cluster among hemodialysis patients in Taiwan. Appl Nurs Res. 2012; 25:190-196.

11. Kopple JD, Shapiro BB, Feroze U, Kim JC, Zhang M, Li Y, Martin DJ. Hemodialysis treatment engenders anxiety and emotional distress. Clin Nephrol. 2017; 88:205-217.

12. Jablonski A. The multidimensional characteristics of symptoms reported by patients on hemodialysis. Nephrol Nurs J. 2007;34:29-37

13. Curtin RB, Bultman DC, Thomas-Hawkins C, Walters BA, Schatell D. Hemodialysis patients' symptom experiences. effects on physical and mental functioning. Nephrol Nurs J. 2002; 29:562, 567-574.

14. Thong MS, van Dijk S, Noordzij M, Boeschoten EW, Krediet RT, Dekker FW, Kaptein AA. Symptom clusters in incident dialysis patients. associations with clinical variables and quality of life. Nephrol Dial Transplant. 2009; 24:225-230.

15. Amro A, Waldum B, von der Lippe N, Brekke FB, Dammen T, Miaskowski C, Os I. Symptom clusters predict mortality among dialysis patients in Norway. a prospective observational cohort study. J Pain Symptom Manage. 2015; 49:27-35.

16. Waltz CF, Strickland O, Lenz ER: Measurement in Nursing and Health 2010, New York, NY. Springer Publishing Company.

17. Hayton JC, Allen DG, Scarpello V. Factor Retention Decisions in ExploratoryFactor. Analysis: a Tutorial on Parallel Analysis. Organizational Research Methods. 2004; 7: 191-205.

18. Polit DF, Beck CT. Essentials of Nursing Research. Methods, Appraisal, and. Utilization (8th ed.). Philadelphia, PA, USA: Lippincott Williams \& Wilkins 2008.

19. Field A: Discovering Statistics Using SPSS for Windows. London, UK: Sag. Publications 2000.

20. Flythe JE, Hilliard T, Lumby E, Castillo G, Orazi J, Abdel-Rahman EM, Pai AB, Rivara MB, St Peter WL, Weisbord SD, et al. Fostering Innovation in Symptom Management among Hemodialysis Patients. Paths Forward for Insomnia, Muscle Cramps, and Fatigue. Clin J Am Soc Nephrol. 2019; 14:150-160. 
21. Palmer SC, Saglimbene V, Mavridis D, Salanti G, Craig JC, Tonelli M, Wiebe N, Strippoli GF. Erythropoiesis-stimulating agents for anaemia in adults with chronic kidney disease. a network metaanalysis. Cochrane Database Syst Rev 2014;12.

22. Lee WC. Gastrointestinal diseases in dialysis patients. Kidney and Dialysis. 2016; 28:185-188.

23. Morfin JA, Fluck RJ, Weinhandl ED, Kansal S, McCullough PA, Komenda P. Intensive Hemodialysis and Treatment Complications and Tolerability. Am J Kidney Dis. 2016; 68:S43-50.

24. Rimmelé T, Kellum JA. Oliguria and fluid overload. Contrib Nephrol. 2010; 164:39-45.

25. Huang YS, Fang CH, Chen YC. Sudden Cardiac Death in Hemodialysis. Kidney and Dialysis. 2017; 29:136-141.

26. Tuncel D, Orhan FO, Sayarlioglu H, Islk IO, Utku, U, Dinc A. Restless legs. syndrome in hemodialysis patients: association with depression and quality of life. Sleep and Breathing. 2011;15: 311-315.

27. Fong CS. Neurological Complications in Uremia. Acta Neurologica Taiwanica. 2008; 17: 117-126.

28. Mettang T, Kremer AE: Uremic pruritus. Kidney Int 2015; 87:685-691.

29. Liu CW, Cheng CY, Chen TH. Medical treatment of secondary. hyperparathyroidism. Kidney and Dialysis. 2015; 27: 135-140.

30. Campese VM, Adenuga G: Electrophysiological and clinical consequences of hyperkalemia. Kidney Int Suppl. 2016; 6:16-19.

31. Hsieh MH, Tsai CW. Phosphate binders in patients with chronic kidney disease: update. Kidney and Dialysis. 2018; 30:170-177.

32. Fong CS. Neurological complications in uremia. Acta Neurol Taiwan. 2008, 17:117-126.

\section{Tables}

Table 1. Exploratory factor analysis (EFA) results and Cronbach's alpha coefficients. 


\begin{tabular}{|c|c|c|c|c|c|}
\hline \multirow[t]{2}{*}{ Symptom } & \multicolumn{4}{|c|}{ Factor loading } & \multirow[t]{2}{*}{ Cronbach's alpha } \\
\hline & Factor 1 & Factor 2 & Factor 3 & Factor 4 Factor 5 & \\
\hline :tor 1 & & & & & 0.89 \\
\hline ədness & 0.87 & & & & \\
\hline :k of vitality & 0.82 & & & & \\
\hline :k of energy & 0.81 & & & & \\
\hline scle weakness & 0.78 & & & & \\
\hline ' mouth/thirst & 0.73 & & & & \\
\hline tigo & 0.63 & & & & \\
\hline adache & 0.62 & & & & \\
\hline :tor 2 & & & & & 0.85 \\
\hline st pain & & 0.84 & & & \\
\hline Irtness of breath & & 0.83 & & & \\
\hline ;pnea & & 0.77 & & & \\
\hline st tightness & & 0.75 & & & \\
\hline hythmia & & 0.65 & & & \\
\hline :k of appetite & & 0.62 & & & \\
\hline :tor 3 & & & & & 0.80 \\
\hline king in night & & & 0.82 & & \\
\hline uble falling asleep & & & 0.78 & & \\
\hline ly skin & & & 0.75 & & \\
\hline :tor 4 & & & & & 0.77 \\
\hline It pain & & & & 0.88 & \\
\hline 'e muscles & & & & 0.80 & \\
\hline nbness & & & & 0.78 & \\
\hline :tor 5 & & & & & 0.73 \\
\hline niting & & & & 0.87 & \\
\hline usea & & & & 0.80 & \\
\hline Imps & & & & 0.66 & \\
\hline al scale & & & & & 0.93 \\
\hline
\end{tabular}

Note. Factor 1 = 'insufficient energy/vitality; Factor 2 = cardiac-pulmonary distress; Factor $3=$ sleep disturbance; Factor $4=$ musculoskeletal distress; Factor $5=$ gastrointestinal distress 